\title{
Number of Slices
}

National Cancer Institute

\section{Source}

National Cancer Institute. Number of Slices. NCI Thesaurus. Code C116125.

The total number of image slices that are recorded during an imaging procedure. 